\title{
Oxidación de pirargirita con metabisulfito y cianuración de plata
}

\author{
I. Hernández Martínez¹, V. González Martínez¹, M. Reyes Pérez" ${ }^{*}$, A.M. Teja Ruiz², \\ M. Pérez Labraํ, J.C. Juárez Tapia ${ }^{1}$, F.R. Barrientos Hernández ${ }^{1}$, G. Reyes Urbano ${ }^{1}$
}

\begin{abstract}
${ }^{1}$ Área Académica de Ciencias de la Tierra y Materiales, Universidad Autónoma del Estado de Hidalgo, Carr. Pachuca-Tulancingo. Km. 4.5, Mineral de la Reforma, Hidalgo, 42184, México, , hernandez_isabel48@hotmail.com,vnt_goma@hotmail.com,miguelabra@hotmail.com, jutj731101@hotmail.com, frbh68@hotmail.com, gurbano2003@yahoo.com.mx ${ }^{2}$ Escuela Superior de Ingeniería Química e Industrias Extractivas, Instituto Politécnico Nacional, Unidad profesional, Adolfo López Mateos. C.P. 07738, México, D.F. ice9791@gmail.com

*Autor de correspondencia: mreyes@uaeh.edu.mx
\end{abstract}

\section{RESUMEN}

La pirargirita $\left(\mathrm{Ag}_{3} \mathrm{SbS}_{3}\right)$ es una sulfosal de plata considerada como refractaria a la cianuración, por tal razón, requiere un pre tratamiento a priori a la lixiviación de plata con cianuro. Este trabajo de investigación estudia el efecto de la concentración y el tiempo de reacción del pre tratamiento con metabisulfito y su posterior lixiviación con ion cianuro. El mineral se caracterizó por técnicas instrumentales de DRX y MEB-EDS se constató la presencia de una única fase mineral de pirargirita. Se encontró que una concentración de $1.05 \mathrm{E}^{-1} \mathrm{M}$ de metabisulfito y 96 horas de pre oxidación, logra un máximo porcentaje de lixiviación del $25 \%$ en un tiempo de 68 horas, la lixiviación se ve favorecida cuando se lleva a cabo el incremento de la concentración del agente oxidante en $2.1 \mathrm{E}^{-1} \mathrm{M}$ y el tiempo de lixiviación, contribuyendo a lograr recuperaciones de plata del 37\%. El análisis por FTIR de los productos sólidos de la lixiviación indican la presencia de fuertes bandas de absorción de los enlaces $\mathrm{Sb}-\mathrm{O}$ del $\mathrm{Sb}_{2} \mathrm{O}_{3}$, estos óxidos son responsables de la refractariedad de la pirargirita a la cianuración, pasivando la superficie y reduciendo los valores de lixiviación de plata por debajo de 50 \%.

Palabras Clave: Pirargirita, Lixiviación, Plata, Metabisulfito, Óxidos de antimonio.

\section{INTRODUCCIÓN}

La pirargirita $\left(\mathrm{Ag}_{3} \mathrm{SbS}_{3}\right)$ es uno de los minerales de plata más importantes, cristaliza en el sistema hexagonal, contiene el $59.7 \%$ de plata $(\mathrm{Ag}), 22.5 \%$ antimonio (Sb) y $18.8 \%$ azufre (S). Ocurre abundantemente en depósitos minerales en zonas mineras como Zacatecas y Guanajuato, México. Es refractario al cianuro y, por lo tanto, la lixiviación de plata en el proceso de cianuración estándar es lenta [1]. En la actualidad, la cianuración es el método más extensamente utilizado en el mundo para lixiviar minetrales preciosos. El agotamiento de los minerales de plata fácilmente lixiviables y el incremento en los precios de la plata han forzado a la industria minera a procesar minerales complejos que pueden ser refractarios al cianuro. Se considera refractario un mineral cuando durante la lixiviación con cianuro se extrae menos del $80 \%$. El cianuro se introdujo industrialmente hace más de un siglo y desde entonces se han usado en varios tipos de aplicaciones mineras, por ejemplo, en el proceso de flotación actúa como depresor de esfalerita [2,3]. 
Debido a que los minerales refractarios de plata y de oro no responden a la cianuración directa, requieren un pretratamiento para liberar al oro y la plata antes de la cianuración para que mejore la extracción [4]. Los métodos que se han practicado industrialmente para incrementar las recuperaciones de metales preciosos son tostación [4], oxidación a presión [5], biooxidación [6] y molienda ultra fina [7]. Otra técnica para incrementar la recuperación de la plata es la pre reacción con cal y nitrato de plomo con la finalidad de pasivar las impurezas minerales, esta técnica incrementa la lixiviación [8].

Se ha demostrado previamente que la lixiviación con sulfuros alcalinos son métodos de pretratamiento efectivo que incrementan la disolución con cianuro extrayendo más plata y oro de los minerales refractarios de antimonio contenido en especies como Zinkenita $\left(\mathrm{Pb}_{4} \mathrm{Sb}_{22} \mathrm{~S}_{42}\right)$ y andurita $\left(\mathrm{Sb}_{2} \mathrm{PbAgS}_{6}\right)$ [9], junto a esto se tiene que el hidróxido de sodio puede disolver antimonio de minerales como estibinita [10]. Por lo tanto, los minerales que presentan refractariedad a ser lixiviados con cianuro son los que contienen antimonio en su estructura, los cuales necesitan un pretratamiento para mejorar la recuperación de oro y plata. Este estudio de investigación evalúa la influencia de un proceso de pretratamiento antes de la lixiviación con cianuro, usando metabisulfito de sodio evaluando la concentración del reactivo de pre tratamiento, el tiempo y la concentración de cianuro.

\section{PARTE EXPERIMENTAL}

Para estudiar la lixiviación de plata del mineral previamente pre-oxidado o pre-tratado se emplearon piezas puras de mineral de pirargirita $\mathrm{Ag}_{3} \mathrm{SbS}_{3}$ proveniente de la región minera de Guanajuato, México. Macroscópicamente se aprecia que se trata de especímenes de alta pureza, no se observan otras fases mineralógicas que contaminen el mineral tal como se aprecia en la Figura 1.

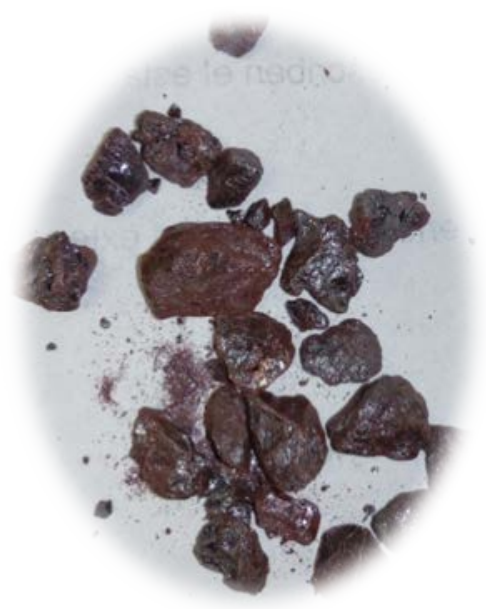

Figura 1. Mineral de pirargirita empleado en esta investigación.

El mineral de pirargirita, se pulverizó en un mortero de ágata, se tamizó en húmedo empleando una serie de tamices Tyler, malla 100, 200, 270, 325, 400 y el fino, con tamaños de partículas de $(180 \mu \mathrm{m}, 75 \mu \mathrm{m}, 53 \mu \mathrm{m}, 43 \mu \mathrm{m}, 37 \mu \mathrm{m}$ y $<37 \mu \mathrm{m}$ respectivamente. El producto tamizado se dejó secar a temperatura ambiente, se 
caracterizó mediante DRX, MEB-EDS. Para la realización de todas las pruebas experimentales se decidió usar el tamaño de partícula de la malla $+325(43 \mu \mathrm{m})$. Para llevar a cabo la experimentación se usó $1 \mathrm{~L}$ de agua desionizada, se colocó en un reactor de vidrio y se agitó mecánicamente con una propela de cuatro aspas fabricada de material inerte (plástico), Posteriormente se adicionan $0.5 \mathrm{~g}$ de pirargirita malla +325 pesados en una balanza de precisión $(0.001 \mathrm{~g})$. Tanto el pH como el ORP se monitorearon durante todas las pruebas, a continuación, en pruebas por separado se adicionó el metabisulfito de sodio $\left(\mathrm{Na}_{2} \mathrm{~S}_{2} \mathrm{O}_{5}\right)$ en concentraciones de $0.052,0.105,0.210 \mathrm{M}$. Los tiempos estudiados de pre-oxidación con metabisulfito fueron; 4, 7, 48, 72, 96 у 120 horas, monitoreando el pH y el ORP, continuamente, el reactor se tapó para evitar en lo mayor posible, pérdidas de volumen por evaporación. Al finalizar cada tiempo de pre tratamiento, el pH se ajustó en alrededor de 9 con hidróxido de sodio, posteriormente se adicionó para cada una de las pruebas, una concentración de cianuro de potasio de 3.07 $\times 10^{-2} \mathrm{M}$, se estudiaron diferentes tiempos de cianuración $68,96,100,180$, y 200 horas.

Durante todo el tiempo de lixiviación con cianuro, se colectaron muestras de la solución, se filtraron y se determinaron vía ICP en su concentración de Ag, Sb, cabe recalcar que el sistema de reacción fue monitoreado todo el tiempo de cianuración, midiendo el pH y el ORP. La relación empleada para determinar el avance de la reacción es:

$$
\% A g=\frac{L * A f o r o * \text { dilución }}{w_{m} * 10000}
$$

Donde L es la lectura obtenida en el ICP en PPM (mg/L), el aforo, es el volumen (ml) de agua total del experimento, la dilución es el factor de dilución el cual de obtiene dividiendo el Volumen del aforo $(\mathrm{ml})$ entre el volumen de la alícuota $(\mathrm{ml}), \mathrm{W}_{\mathrm{m}}$ es el peso de muestra en gramos, y 10000 es el factor de conversión de las unidades de esta relación. Los sólidos residuales de las pruebas se lixiviación se recuperaron, se lavaron con abundante agua y se caracterizaron vía espectroscopia de infrarrojo por transformada de Fourier FTIR y microscopia electrónica de barrido MEB en conjunto con micro análisis por dispersión de energias EDS.

\section{RESULTADOS}

La Figura 2 muestra el espectro de difracción de rayos X (DRX) del mineral, el cual se indexó con la base de datos del software Match, se encontró que la pirargirita es la única fase mineralógica cristalina presente, identificada con el patrón de difracción PDF 00019-1135. El análisis por MEB y mapeos elementales de la pirargirita se muestra en la Figura 3. Se observa que contiene los elementos característicos de esta fase mineral en algunas de las pruebas de detecto la presencia de cobre, el cual debido a su menor porcentaje no es posible detectar por difracción de rayos $X$. 


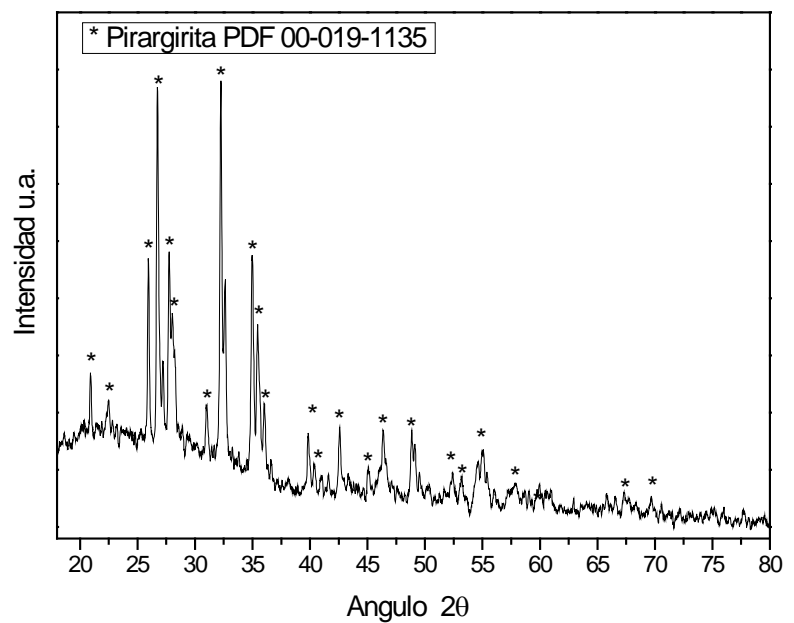

Figura 2. Espectro de difracción de rayos $X(D R X)$ del mineral de pirargirita
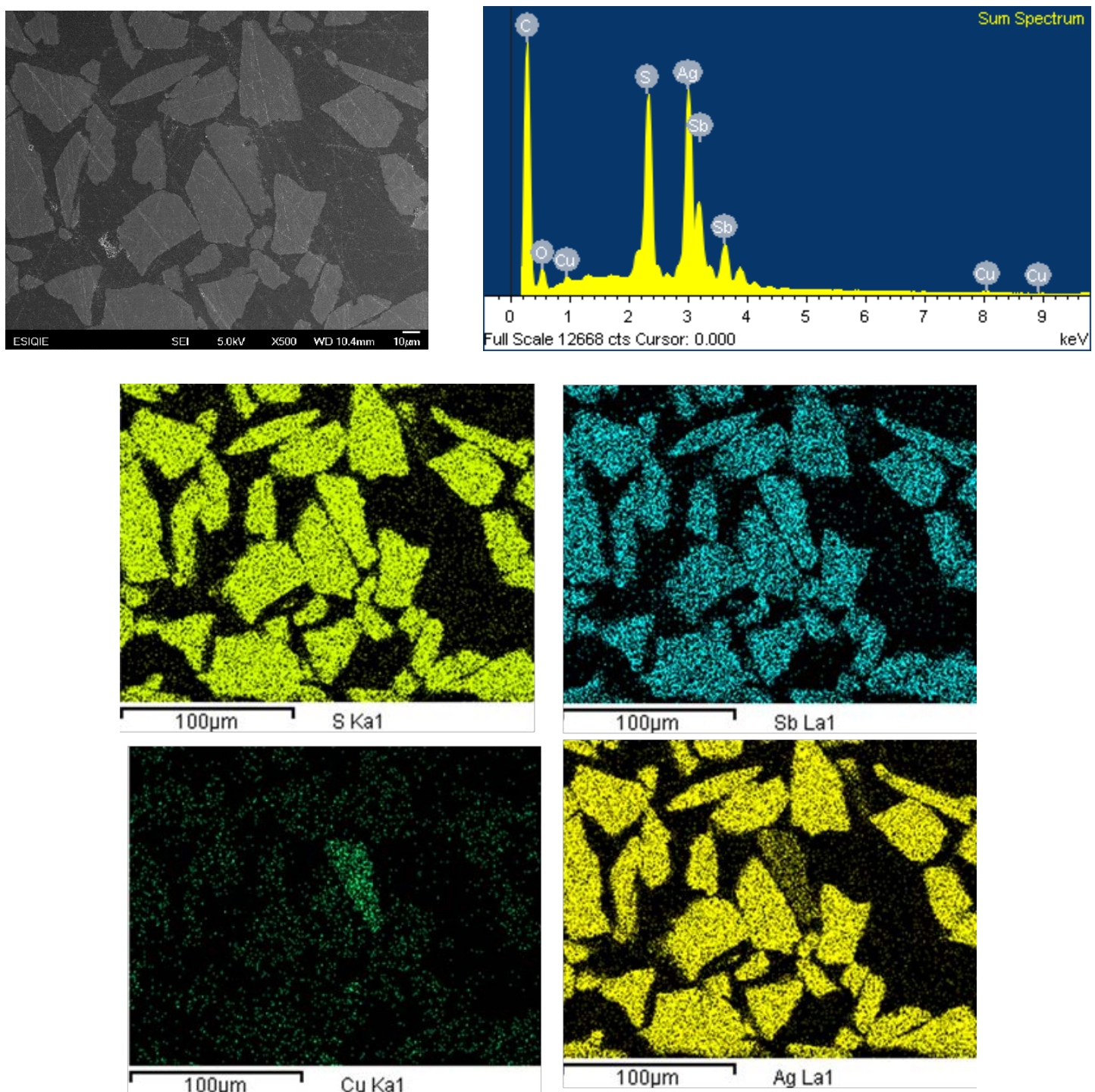

Figura 3. Micrografías de MEB y mapeos elementales del mineral de pirargirita 
La Tabla 1 muestra el microanálisis semi cuantitativo por dispersión de energías del mineral de pirargirita, donde el elemento mayoritario es la plata, lo cual la hace una importante mena de este metal valioso sin embargo el elevado contenido de antimonio imposibilita la lixiviación de plata por procesos tradicionales, lo cual lleva necesariamente involucrar una etapa previa de oxidación para mejorar la cianuración de plata.

Tabla 1 Análisis semi cuantitativo del mineral de pirargirita

\begin{tabular}{|c|c|}
\hline Elemento & Porcentaje \\
\hline Azufre & 16.25 \\
\hline Oxigeno & 7.90 \\
\hline Cobre & 2.19 \\
\hline Plata & 52.78 \\
\hline Antimonio & 20.89 \\
\hline
\end{tabular}

Los resultados de las pruebas de pre oxidación y lixiviación de plata con cianuro se muestran en la Figura 4, en general, se observa que el porcentaje de extracción incrementa constantemente con el tiempo de reacción. Se esperarían mejores

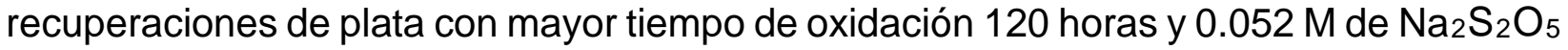
( $2.5 \%$ de extracción de Ag), sin embargo, para la misma concentración de metabisulfito y 68 horas de lixiviación con una concentración de cianuro de 0.037 M la recuperación de plata del mineral oxidado es significativamente mayor, obteniendo alrededor del $25 \%$. La reacción de cianuración esperada seria como indica la ecuación 2. Por otra parte,

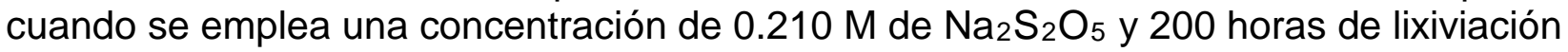
se consigue la máxima extracción de plata en alrededor del $37 \%$.

$$
2 \mathrm{Ag}^{\circ}+4 \mathrm{CN}^{-}+2 \mathrm{H}_{2} \mathrm{O}+\mathrm{O}_{2}=2\left[\mathrm{Ag}(\mathrm{CN})_{2}\right]^{-}+\mathrm{H}_{2} \mathrm{O}_{2}+2 \mathrm{OH}^{-}
$$

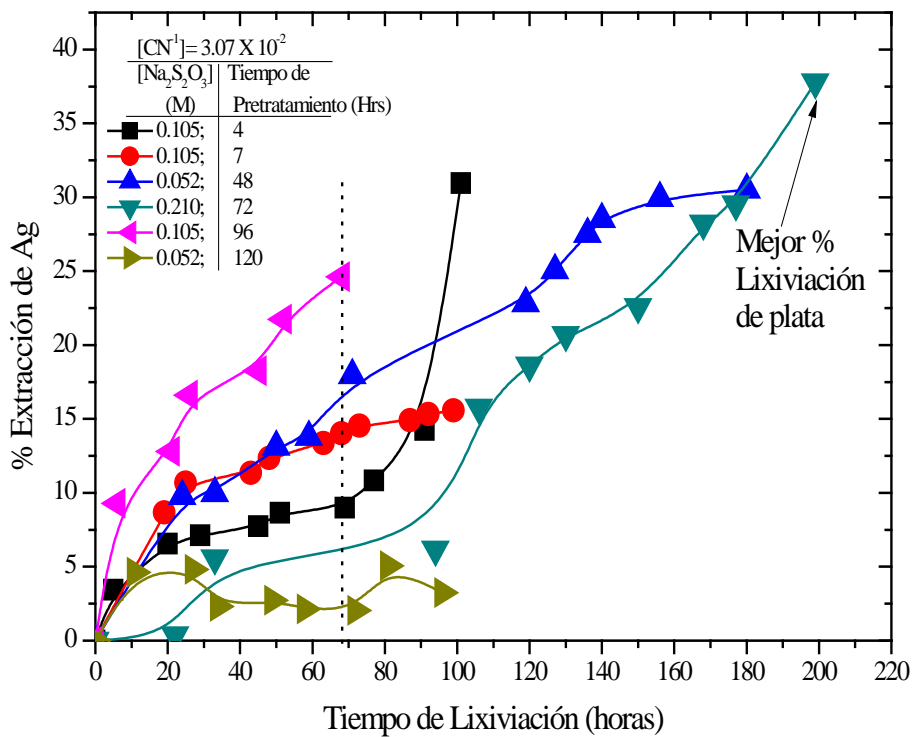

Figura 4. \% Extracción de Plata Vs. Tiempo de lixiviación (horas) para las diferentes concentraciones de metabisulfito y tiempos de pre oxidación 
Del monitoreo del $\mathrm{pH}$ y del ORP realizados durante todas las pruebas, se tiene que, con el incremento del tiempo de lixiviación el $\mathrm{pH}$ de la pulpa tiende a disminuir, esto es debido a la lixiviación del resto de los componentes de la pirargirita, tal como el azufre el cual forma ion sulfato $\mathrm{SO}_{4}{ }^{2-}$ en solución disminuyendo el $\mathrm{pH}$, además, del análisis de antimonio realizado vía ICP se detectó que se llega a solubilizar hasta alrededor del 20 $\%$.

En lo que respecta al ORP, este disminuye su carácter reductor es decir su valor negativo es menor con el aumento del tiempo de lixiviación, debido a la naturaleza de las especies formadas durante la cianuración. La Figura 5 muestra la micrografía de MEB - EDS y el mapeo elemental de los polvos residuales, 180 horas de lixiviación con cianuro pre oxidados con $0.052 \mathrm{M}$ de Metabisulfito de sodio. Tal como se mostró en la figura 4 la extracción de plata no es total por tal razón en el mapeo elemental se detectan tanto de azufre, plata y antimonio, este presenta un fenómeno de zonas de mayor concentración generalmente entorno a las superficies de pirargirita.

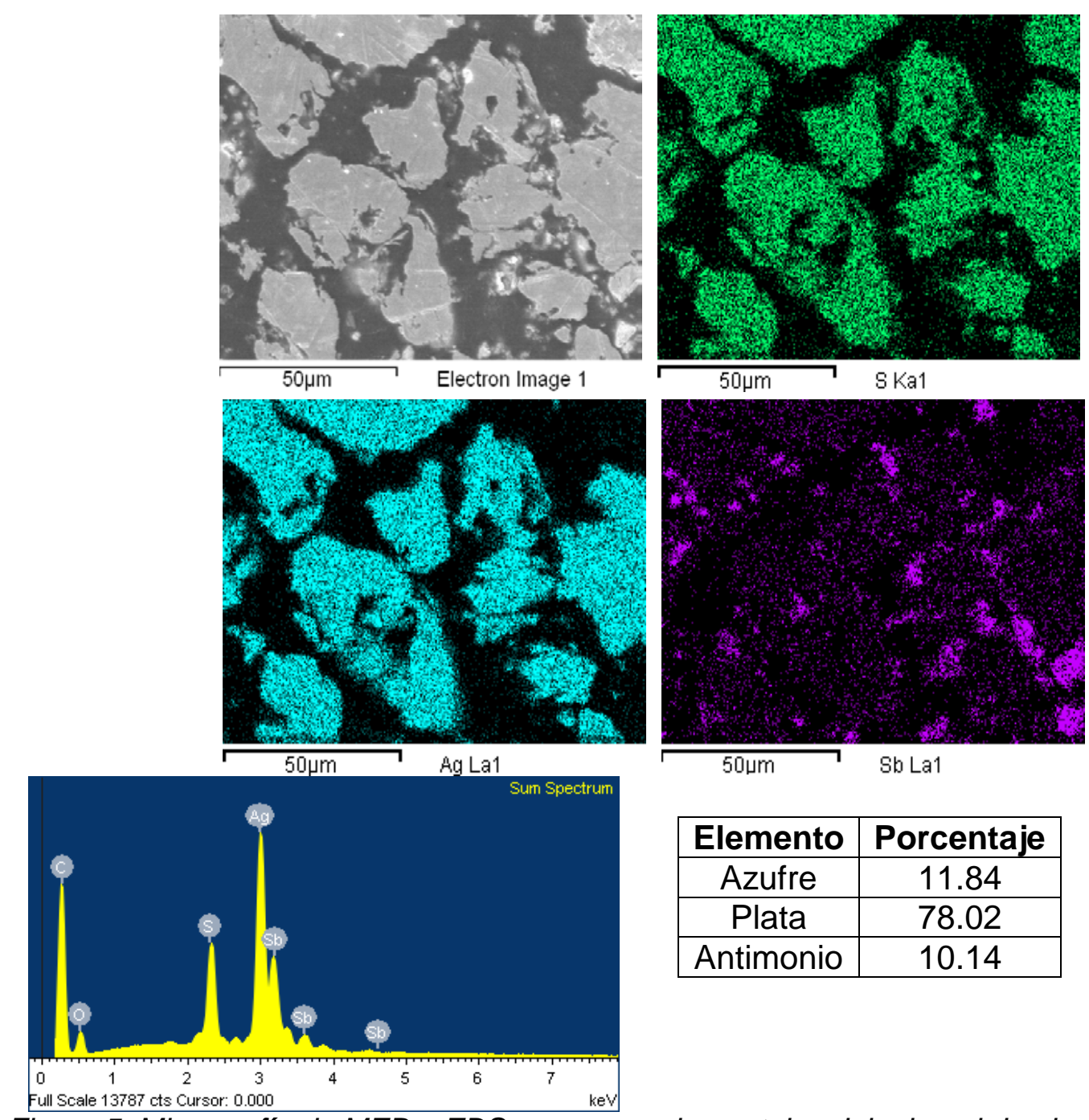

Figura 5. Micrografía de MEB - EDS y mapeos elementales del mineral de pirargirita. Lixiviada $180 \mathrm{~h}$ con $3.02 \times 10^{-2} \mathrm{M}$ de cianuro, pre oxidada con $0.052 \mathrm{M}$ de $\mathrm{Na}_{2} \mathrm{~S}_{2} \mathrm{O}_{5}$. 
La Figura 6 presenta el análisis mediante espectroscopia de infrarrojo por transformada de Fourier de los polvos residuales de la lixiviación de pirargirita por $180 \mathrm{~h}$ con $3.07 \mathrm{X}$ $10^{-2} \mathrm{M}$ de cianuro y pre oxidada por $48 \mathrm{~h}$ con $0.210 \mathrm{M}$ de $\mathrm{Na}_{2} \mathrm{~S}_{2} \mathrm{O}_{5}$, así como el espectro de pirargirita sin oxidar y el óxido de antimonio $\mathrm{Sb}_{2} \mathrm{O}_{3}$. Cuando la pirargirita permanece sin oxidar, se forma en la región espectral del ion sulfato, la escisión de la banda principal con tres bandas de absorción donde el sulfato se enlaza de manera monodentada.

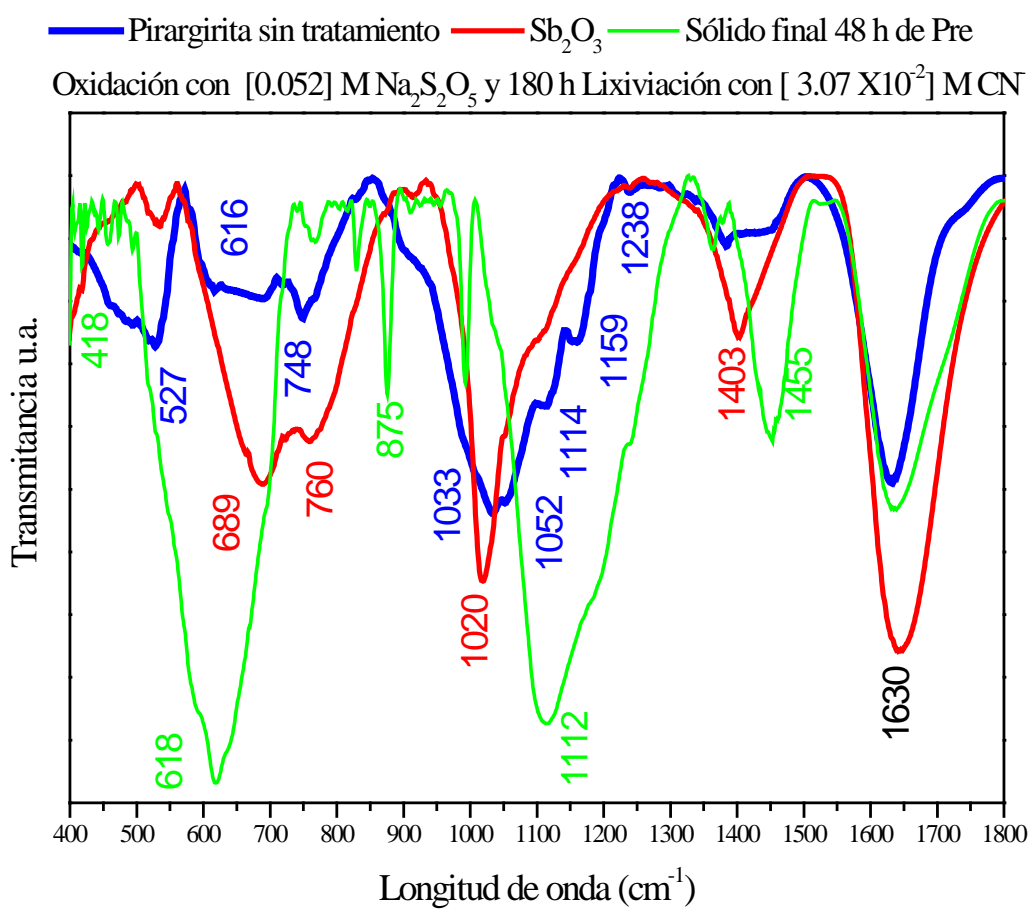

Figura 6. Espectro de infrarrojo de la pirargirita sin tratamiento, polvos finales del proceso de lixiviación y reactivo químico de trióxido de antimonio $\mathrm{Sb}_{2} \mathrm{O}_{3}$

Las bandas de absorción en $618 \mathrm{~cm}^{-1}, 875 \mathrm{~cm}^{-1}, 988 \mathrm{~cm}^{-1}, 1112 \mathrm{~cm}^{-1}$ y 1455 corresponden a los enlaces $\mathrm{Sb}-\mathrm{O}$; lo cual confirma la fuerte presencia de estos óxidos de antimonio los cuales afectan el éxito de la lixiviación generando una capa de difícil acceso para llevar a cabo la extracción de plata.

\section{CONCLUSIONES}

Mediante las técnicas de MEB-EDS y DRX se constató que el mineral estudiado corresponde a una pirargirita $\mathrm{Ag}_{3} \mathrm{SbS}_{3}$ y fue la única fase cristalina detectada por DRX indexada con el patrón de difracción PDF 00-019-1135. El mejor porcentaje de lixiviación de pirargirita se logra cuando se pre oxida por 68 horas con $0.210 \mathrm{M}$ de metabisulfito y una cianuración de 200 horas con $3.07 \times 10^{-2} \mathrm{M}$, el incremento de la concentración de metabisulfito y el tiempo de pre tratamiento no contribuye a lograr mejores recuperaciones. Las bajas recuperaciones se atribuyen a la formación de especies de óxidos de antimonio detectados por FTIR, entorno a la superficie formando una especie refractaria al paso del cianuro este óxido $\mathrm{Sb}_{2} \mathrm{O}_{3}$ pasiva la superficie del mineral e impide que se efectúe la cianuración exitosamente. 


\section{AGRADECIMIENTOS}

A la Universidad Autónoma del Estado de Hidalgo, al Programa para el Desarrollo Profesional Docente PRODEP, y al Instituto Politécnico Nacional unidad ESIQUIE.

\section{BIBLIOGRAFÍA}

[1]. C. Rodríguez Rodríguez, F. Nava Alonso, A. Uribe Salas. Hydrometallurgy 149 (2014) 168-176.

[2]. M.J. Logsdon, K. Hagelstein, T.I. Mudder. International Council on Metals and the Environment (1999) 1-37.

[3]. G. Bao, P. Yongjun, R. Espinosa Gómez. Miner. Eng. 66 (2014) 25-32.

[4]. S.R. La Brooy, H.G. Linge, G.S. Walker. Miner. Eng. 7 (1994) 1213-1241.

[5]. F.P. Gunyanga, T. Mahlangu, R.J. Roman, J. Mungoshi, K. Mbeve. Miner. Eng. 8 (1999) 863-875.

[6]. E. Ciftci, Mineralogy, paragenetic sequence, geochemistry and genesis of the gold and silver bearing upper cretaceous mineral deposits, Northeastern Turkey, PhD Thesis, University of Missouri-Rolla, Faculty of the Graduate School, Geology and Geophysics (2000)

[7]. I.J. Corrans, J.E. Angove. Miner. Eng. 11 (1991) 763-776.

[8]. J. Rajala, G. Deschênes, A. Romanov, G. Kolosay. 2011. CIM J. 2 (2011) 79-84.

[9]. A. İbrahim, C. Oktay, P. Doğan, T. Yves. Hydrometallurgy 146 (2014) 64-71.

[10]. P. Raschman, E. Sminčáková. Hydrometallurgy 113 (2011) 60-66. 